\title{
Article \\ A Systematic Review and Meta-Analysis: Lactobacillus acidophilus for Treating Acute Gastroenteritis in Children
}

\author{
Haixin Cheng ${ }^{1}$, Yi Ma ${ }^{2}$, Xiaohui Liu ${ }^{3}$, Chao Tian ${ }^{3}$, Xuli Zhong ${ }^{1, *}$ and Libo Zhao ${ }^{2, *}$ (D) \\ 1 Department of Pharmacy, Children's Hospital of Capital Institute of Pediatrics, Beijing 100020, China; \\ chhaixin@163.com \\ 2 Department of Pharmacy, Peking University Third Hospital, Beijing 100191, China; mayi@bjmu.edu.cn \\ 3 Department of Pharmacy, Beijing Children's Hospital, Capital Medical University, Beijing 100045, China; \\ liuxiaohui5077@163.com (X.L.); 18811213680@163.com (C.T.) \\ * Correspondence: xuli_zh@163.com (X.Z.); libozhao2011@163.com (L.Z.)
}

Citation: Cheng, H.; Ma, Y.; Liu, X.; Tian, C.; Zhong, X.; Zhao, L. A Systematic Review and Meta-Analysis: Lactobacillus acidophilus for Treating Acute Gastroenteritis in Children Nutrients 2022, 14, 682. https:// doi.org/10.3390/nu14030682

Academic Editor: Ruggiero Francavilla

Received: 17 December 2021

Accepted: 2 February 2022

Published: 6 February 2022

Publisher's Note: MDPI stays neutral with regard to jurisdictional claims in published maps and institutional affiliations.

Copyright: (C) 2022 by the authors. Licensee MDPI, Basel, Switzerland. This article is an open access article distributed under the terms and conditions of the Creative Commons Attribution (CC BY) license (https:// creativecommons.org/licenses/by/ $4.0 /)$.

\begin{abstract}
The efficacy of probiotic strains of Lactobacillus acidophilus to manage acute gastroenteritis in children is still not established. We searched the Cochrane Library, PubMed, EMBASE, and three Chinese literature databases (CNKI, WanFang, and CBM) from their inception to February 2021 for RCTs that compared the use of Lactobacillus acidophilus with no Lactobacillus acidophilus. The grey literature was searched through Google Scholar. Authors of the original papers were contacted for additional data. The study included a total of 15 RCTs involving 1765 patients. Compared with placebo or no treatment, Lactobacillus acidophilus was associated with a reduced duration of diarrhea (moderate quality of evidence), but the effect was not statistically significant when only the individual probiotic strain was provided. Lactobacillus acidophilus was effective when used at a daily dose $\geq 10^{9} \mathrm{CFU}$. There was no difference in the effect of Lactobacillus acidophilus on diarrhea duration among Asian, European, or American countries. Lactobacillus acidophilus reduced the frequency of diarrhea on day 2 to day 5 . However, it was statistically significant on day 3. When administered at a dosage of more than $10^{9}$ CFU to children with acute gastroenteritis, moderate- to low-quality data showed that Lactobacillus acidophilus reduced the duration of diarrhea and conferred a benefit for frequency of diarrhea.
\end{abstract}

Keywords: Lactobacillus acidophilus; acute gastroenteritis; children; systematic review; meta-analysis

\section{Introduction}

For children under the age of five, acute gastroenteritis (a clinical illness characterized by increased stool frequency, accompanied with or without vomiting, fever, or stomach discomfort) ranks second on the list of main causes of mortality in the world [1]. Despite a 90 percent reduction in diarrhea-related mortality over the previous forty years, acute gastroenteritis continues to be a serious public health concern. For example, in 2018, approximately 500,000 children died from diarrhea [2]. The European Evidence-Based Guidelines for managing acute gastroenteritis in children concluded that heat-killed Lactobacillus acidophilus LB (low quality of evidence, weak recommendation) demonstrated some efficacy in reducing acute gastroenteritis-related symptoms in pediatric age groups [3]. Lactobacillus acidophilus achieved maximum survival rates and $\mathrm{CFU} / \mathrm{mL}$ after adaptation at $\mathrm{pH} 4.2$ and 5.0 [4]; in consequence, it can easily reach the small intestine $(\mathrm{pH}<7)$, and is able to thrive in the small intestine rather than the colon $(\mathrm{pH}>7)$. Possible mechanisms for the therapeutic effects of Lactobacillus acidophilus include amelioration of the impairment of electrolyte absorption [5], maintenance of immunological homeostasis [6], antibiotic activity/antiviral activity [7], and promotion of intestinal epithelial integrity [8]. In addition, in 2018, a network meta-analysis of randomized and quasi-randomized trials found high-quality evidence that combinations of Lactobacillus acidophilus and other probiotic strains could reduce the duration of diarrhea by approximately one day (MD $-26.3 \mathrm{~h}, 95 \% \mathrm{CI}-16.2$ to -36.1) compared with standard care or placebo [9]. 
However, in the light of recent null studies, there is still controversy as to whether the efficacy of Lactobacillus acidophilus has been established. A relatively large, prospective randomized controlled trial (RCT) carried out in Vietnam, including 290 children aged 11.8 months to 21.5 months, compared the operation of Lactobacillus acidophilus, at a dose of $4 \times 10^{8}$ CFU twice daily, to a matching placebo, found no differences in outcomes, including duration of diarrhea and stool frequency in the first three days after enrollment, between groups [10]. Moreover, in several additional randomized controlled trials (RCTs), no difference in diarrhea duration was reported across groups [11,12]. The purpose of this study was to assess the effectiveness of Lactobacillus acidophilus supplementation for treating children with acute gastroenteritis, and to give suggestions on probiotic treatments for acute gastroenteritis in children according to the assessment result.

\section{Materials and Methods}

\subsection{Study Design}

The study protocol was designed by the authors H.C., Y.M. and L.Z. This report complies with the recommendations of the Preferred Reporting Items for Systematic Reviews and Meta-Analyses (PRISMA: http://www.prisma-statement.org/ (accessed on 21 February 2021)) guidance. The systematic review and meta-analysis protocol were registered with the PROSPERO (National Institute for Health Research, NIHR. http://www.crd.york.ac.uk/prospero/ (accessed on 21 February 2021)) international prospective register of systematic reviews (CRD42021254066).

\subsection{Inclusion and Exclusion Criteria}

All relevant RCTs that compared use of Lactobacillus acidophilus (as a single ingredient or a multispecies synbiotic mixture, in all formulations, at any dose, regardless of the strain manufacturer), with no Lactobacillus acidophilus (defined as placebo or no treatment), were eligible for inclusion. Patients were children ( $<18$ years old) with acute gastroenteritis. Only RCTs published in English and Chinese were considered. The primary outcome measure was the duration of diarrhea. The secondary outcome measure was the frequency of diarrhea at various time intervals. Letters to the editor, abstracts and scientific conference proceedings were all omitted.

\subsection{Search Strategy}

To acquire relevant evidence, the Cochrane Library, PubMed, EMBASE and three Chinese literature databases (CNKI, WanFang, and CBM) were searched from their inception to February 2021. The principal search terms and keywords used were as follows: diarrhea/diarrhoea, diarrh*, gastroenteritis, probiotic* , Lactobacillus acidophilus, and randomized controlled trial. For full details, see Table S1. Additionally, the grey literature was searched through Google Scholar. Some clinical trials databases (https://www. ClinicalTrials.gov (accessed on 21 February 2021) and https: / /www.ClinicalTrialsRegister. eu (accessed on 22 February 2021)) were also searched for RCTs. The reference lists from identified studies and key review articles were also searched if the title, abstract, name of substance word, subject heading word, keyword heading word, protocol supplementary concept word or unique identifier contained all of "Lactobacillus acidophilus" and "acute gastroenteritis" and "children". If further information was required, the authors of the original papers were contacted for additional data.

\subsection{Data Extraction}

Study inclusion was determined by two investigators working separately, with the senior investigator addressing any discrepancies between the two investigators. The same investigators independently extracted data on study design using a standardized data extraction form. A data extraction form was developed to facilitate the electronic comparison of entry. The following data were extracted: study characteristics (including author, year of publication, percentage of patient follow-up, country, research foundation), 
patient characteristics (mean age), interventions characteristics (drug groups, intervention doses, and intervention duration).

\subsection{Quality Assessment and Risk of Bias}

The GRADE (Grading of Recommendations, Assessment Development and Evaluations) approach, supported by an electronic tool (McMaster University and Evidence Prime. https:/ /gdt.gradepro.org (accessed on 21 February 2021)) [13], was used in the evidence quality assessment for the primary outcome-duration of diarrhea, and the secondary outcome-frequency of diarrhea on specific days. The quality of evidence (also known as certainty of the evidence) was categorized into four categories based on the likelihood of bias, the directness of evidence, and the consistency and accuracy of estimates: high, moderate, low, and extremely low.

The risk of bias was assessed using the revised Cochrane risk-of-bias tool. The riskof-bias parameters included the type of randomization process (selection bias), allocation concealment (selection bias), blinding of participants and personnel (performance bias), blinding of outcome assessment (detection bias), and incomplete outcome data (attrition bias). Furthermore, biases, such as selective reporting (reporting bias) and other forms of prejudice, were taken into consideration. Due to a lack of available information, an item was classified as having an ambiguous risk of bias if it could not be assessed [14].

\subsection{Statistical Analysis}

The mean difference (MD) and 95\% confidence interval (95\% CI) were used as the metrics of choice for treatment effects with random-effects models. Pooled data was assessed using available case analysis. Data were analyzed for every participant for whom the outcome was obtained, rather than intention-to-treat analysis with imputation. The presence of heterogeneity among studies was measured with $\chi^{2}$ test, with a $p$-value of up to 0.10 considered significant. To measure consistency, we used the $\mathrm{I}^{2}$ test. An $\mathrm{I}^{2}$ value of $0 \%$ indicates that there has been no observed heterogeneity, while rising values imply growing heterogeneity in the data. $\mathrm{I}^{2}$ values above $50 \%$ suggest a significant degree of heterogeneity.

When at least ten randomized controlled trials were available, funnel plots were used to examine reporting biases for the primary outcome. The presence of reporting bias was investigated with Begg and Egger tests [15]; $p<0.05$ implies publication bias. This was accomplished via the use of funnel plots and other visual estimation techniques. Statistical analyses were performed using STATA software (Version 15.1).

The data were analyzed using Review Manager (Version 5.4. the Nordic Cochrane Centre, the Cochrane Collaboration, Copenhagen, Denmark, 2020). For change in duration of diarrhea, the mean duration was used as reported. When reported, the standard deviation (SD) was utilized or computed from the median and interquartile range (IQR). In four trials reporting median and IQR $[10,11,16,17]$, missing means and SDs were estimated using the formula recommended by Wan et al. [18] and McGrath et al. [19].

Four separate pre-specified subgroup analyses were performed for the primary outcomes according to the presence of potential trial-level effect modifiers:

1. Probiotic species (individual probiotic strains Lactobacillus acidophilus vs. Lactobacillus acidophilus + other probiotic strains)

2. The daily dose of Lactobacillus acidophilus (high dose $\left[\geq 10^{10} \mathrm{CFU} /\right.$ day $]$ vs. $\left[10^{9} \sim 10^{10} \mathrm{CFU} /\right.$ day $]$ vs. low dose $\left[<10^{10} \mathrm{CFU} /\right.$ day $]$ )

3. Setting (studies carried out in geographical areas, Asia vs. Europe vs. America)

4. Etiology of diarrhea (rotavirus-positive $100 \%$ vs. rotavirus-positive $1 \sim 99 \%$ vs. unknown etiology).

\section{Results}

3.1. Search Results

For a flow diagram documenting the identification process for eligible trials, see Figure 1. Detailed characteristics of the included RCTs are presented in Table S2, and excluded 
trials are shown in Table S3. Ultimately, 15 RCTs that randomized 1765 participants (895 in the experimental group and 870 in the control group) were included [10-12,16,17,20-29].

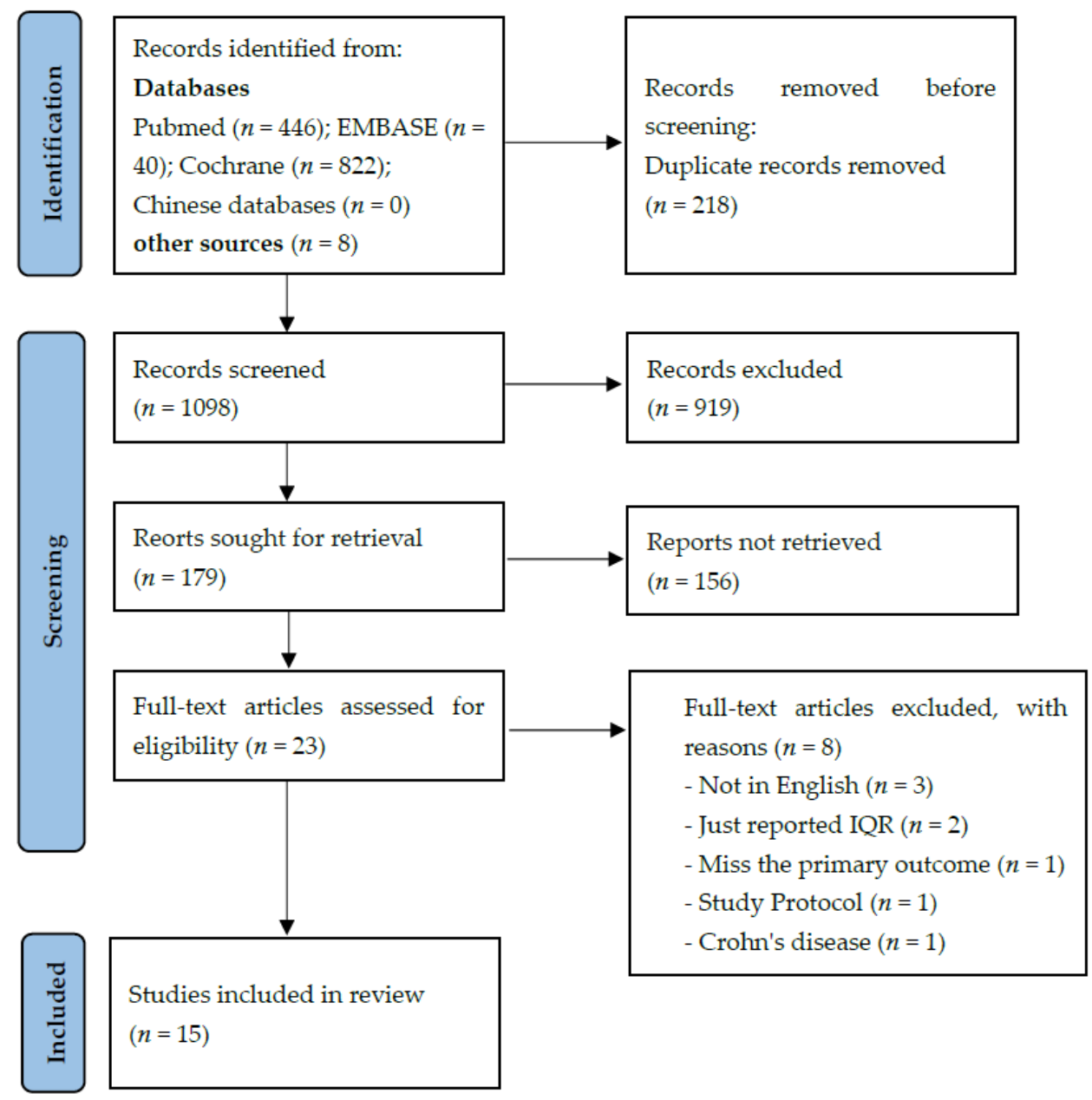

Figure 1. Flow diagram of search strategy and study selection.

\subsection{Characteristics of Included Studies}

The participants in the studies varied in age from 1 month to 12 years. The number of people that took part in the trials varied from 46 to 290 . Included trials were carried out in Thailand [27], India [29], Peru [16], Iran [19,25], Vietnam [10], TaiWan [23], Israel [24], France [28], Belgium [17,26], Turkey [21], Indonesia [11], Korea [12], and China [22]. All the research studies considered were single-center trials.

The most common administered daily dose of Lactobacillus acidophilus ranged from $10^{9}$ to $10^{10} \mathrm{CFU}[12,17,21,23,24,26]$. Other daily doses were $<10^{9} \mathrm{CFU}[10,11]$, and $\geq 10^{10} \mathrm{CFU}[16,25,27-29]$. The doses were unknown in two trials. The duration of the intervention was inconsistent, lasting 2 days [27], 3 days [12,28,29], 5 days [10,21], 7 days [11,17,26], or for an unspecified period [16,19,22-25].

The blank contrast group were placebo in 6 trials and no Lactobacillus acidophilus in the remaining trials. In all studies, Lactobacillus acidophilus was used in addition to rehydration therapy, consisting of an oral rehydration solution and/or intravenous rehydration.

\subsection{Assessment of Risk of Bias and Publication Bias}

Most trials were at risk of bias for at least one of the domains. The appendix presents the findings of the risk-of-bias evaluation (see Figure S1). Only 8 (53\%) of the trials adequately generated their randomization sequence, while just $11(73 \%)$ adequately concealed allocation, and $12(80 \%)$ trials blinded all involved parties (e.g., participants, study per- 
sonnel and outcome assessors). A total of 14 trials (93\% of all trials) provided complete outcome data, defined as at least $80 \%$ follow-up. Only 5 trials were considered at low risk for overall risk of bias.

GRADE analysis for the selected outcomes is presented in Table S4.

High levels of statistical heterogeneity $\left(\mathrm{I}^{2} \geq 50 \%\right)$ were found for both the primary outcome (the duration of diarrhea $\left[\mathrm{I}^{2}=94 \%\right]$ ) and the secondary outcome (the frequency of diarrhea at various times interval $\left.\left[\mathrm{I}^{2}=84 \%\right]\right)$. Publication bias was formally evaluated only in the analysis of the duration of diarrhea. There was publication bias for this outcome (Egger test's test $p=0.015$; see Figure S2). Because of the limited number of papers included in the analysis $(<10)$, publication bias was not officially examined using a funnel plot for other outcomes.

\subsection{Results of Included Studies}

A summary of the results is presented in Table 1.

Table 1. Overview of the results.

\begin{tabular}{|c|c|c|c|c|c|}
\hline Outcome or Subgroup & $\begin{array}{l}\mathrm{RCT} \\
(n)\end{array}$ & $\begin{array}{l}\text { Participants } \\
\text { (n) }\end{array}$ & $\begin{array}{l}\text { Statistical Method, } \\
\text { Random Effect } \\
\text { Model }\end{array}$ & $\begin{array}{l}\text { Effect Estimate } \\
(95 \% \text { CI })\end{array}$ & $\begin{array}{l}\text { Heterogeneity, } \\
I^{2}(\%)\end{array}$ \\
\hline Duration of diarrhea (days) & 15 & 1765 & MD & $-0.69[-1.04,-0.33]$ & $94 \%$ \\
\hline \multicolumn{6}{|l|}{ Probiotic strains } \\
\hline individual probiotic strains Lactobacillus acidophilus & 6 & 698 & MD & $-0.47[-0.95,0.01]$ & $94 \%$ \\
\hline Lactobacillus acidophilus + other probiotic strains & 9 & 1067 & MD & $-0.91[-1.23,-0.59]$ & $66 \%$ \\
\hline \multicolumn{6}{|l|}{ Daily dose of Lactobacillus acidophilus in all studies } \\
\hline$\geq 10^{10} \mathrm{CFU}$ & 5 & 408 & MD & $-0.69[-1.25,-0.14]$ & $95 \%$ \\
\hline $10^{9} \sim 10^{10} \mathrm{CFU}$ & 6 & 588 & MD & $-0.89[-1.36,-0.42]$ & $78 \%$ \\
\hline$<10^{9} \mathrm{CFU}$ & 2 & 402 & MD & $0.55[0.20,0.91]$ & $0 \%$ \\
\hline \multicolumn{6}{|c|}{ Daily dose of Lactobacillus acidophilus in individual probiotic strains studies } \\
\hline$\geq 10^{9} \mathrm{CFU}$ & 5 & 408 & MD & $-0.69[-1.25,-0.14]$ & $95 \%$ \\
\hline$<10^{9} \mathrm{CFU}$ & 1 & 290 & MD & $0.56[0.20,0.92]$ & $\mathrm{N} / \mathrm{A}$ \\
\hline \multicolumn{6}{|l|}{ Setting } \\
\hline Asia & 11 & 1451 & MD & $-0.66[-1.07,-0.26]$ & $94 \%$ \\
\hline Europe & 3 & 237 & MD & $-0.88[-1.41,-0.36]$ & $43 \%$ \\
\hline America & 1 & 77 & MD & $-0.65[-1.33,0.03]$ & $\mathrm{N} / \mathrm{A}$ \\
\hline \multicolumn{6}{|l|}{ Etiology } \\
\hline Rotavirus-positive $100 \%$ & 1 & 57 & MD & $-1.23[-1.88,-0.58]$ & $\mathrm{N} / \mathrm{A}$ \\
\hline Rotavirus-positive 1\% 99\% & 6 & 718 & MD & $-0.79[-1.43,-0.15]$ & $94 \%$ \\
\hline Unknown etiology & 8 & 990 & MD & $-0.91[-1.24,-0.58]$ & $59 \%$ \\
\hline \multicolumn{6}{|l|}{ Frequency of diarrhea } \\
\hline On day 1 & 6 & 694 & MD & $-0.47[-1.71,0.78]$ & $92 \%$ \\
\hline On day 2 & 7 & 965 & MD & $-0.45[-1.22,0.33]$ & $79 \%$ \\
\hline On day 3 & 4 & 594 & MD & $-0.61[-1.00,-0.23]$ & $64 \%$ \\
\hline On day 4 & 3 & 493 & MD & $-0.23[-0.61,0.16]$ & $0 \%$ \\
\hline On day 5 & 1 & 209 & MD & $0.15[-0.22,0.52]$ & $\mathrm{N} / \mathrm{A}$ \\
\hline
\end{tabular}

Abbreviations: RCT, randomized controlled trial; $95 \%$ CI, 95\% confidence interval; MD, mean difference; N/A not applicable.

\subsubsection{Reporting of Diarrhea Duration}

The primary outcomes are summarized in Figure 2. After conducting 15 trials with 1765 participants, researchers discovered that those who received Lactobacillus acidophilus had shorter diarrhea duration than those who were treated with placebo or received no treatment (MD -0.69 days, $95 \% \mathrm{CI}-1.04$ to -0.33 ; high heterogeneity $\left[\mathrm{I}^{2}=94 \%\right]$ ). 
As intended, all the pre-planned subgroup analyses were performed.

- Probiotic species

There appeared to be a decrease in the duration of diarrhea in children treated both in the individual probiotic strain Lactobacillus acidophilus subgroup (6 RCTs, $n=698$, MD -0.47 days, $95 \% \mathrm{CI}-0.95$ to 0.01 ) and in the Lactobacillus acidophilus + other probiotic strains subgroup ( 9 RCTs, $n=1067$, MD -0.91 days, $95 \%$ CI -1.23 to -0.59 ); however, the former effect was not statistically significance between the probiotic group and the placebo group (Figure 2). The test for subgroup differences suggested that there was no significant difference $(p=0.14)$, meaning that combined other probiotic strains may not modify the treatment effect. However, a smaller group of trials and participants contributed to the Lactobacillus acidophilus subgroup than to the Lactobacillus acidophilus + other probiotic strains subgroup. There was also heterogeneity between the trials within each subgroup. Overall, whether there is no efficacy of Lactobacillus acidophilus, or whether there is an actual significant subgroup effect, requires further investigation. The subdivided probiotic mixture was analyzed by subgroup (Figure S3).

\section{- $\quad$ Dose}

Lactobacillus acidophilus was effective when used at a daily dose $\geq 10^{10} \mathrm{CFU}$ (5 RCTs, $n=408$, MD -0.69 days, $95 \%$ CI -1.25 to -0.14 ) and $10^{9} \sim 10^{10}$ CFU (6 RCTs, $n=588$, $\mathrm{MD}-0.89$ days, $95 \% \mathrm{CI}-1.36$ to -0.42$)$; the latter dosage produced results of more significance (Figure 3). However, there was no reduction nor increment in the duration of diarrhea when used at a daily dose $<10^{9} \mathrm{CFU}$ ( 2 RCTs, $n=402$, MD 0.55 days, $95 \%$ CI 0.20 to 0.91$)$. The test for subgroup differences suggests a significant difference $(p<0.00001)$, meaning that the daily dose of probiotic may modify the treatment effect. Nevertheless, a smaller group of trials and participants contributed to the $<10^{9} \mathrm{CFU}$ subgroup (2 RCTs, $n=402$ ) than to the $\geq 10^{9} \mathrm{CFU}$ subgroup (11 RCTs, $n=988$ ), indicating that the analysis may expand the differences between subgroups. The same result occurred in the individual probiotic strain Lactobacillus acidophilus (Figure S4).

\section{- $\quad$ Setting}

There appeared to be a reduction in the duration of diarrhea in children, treated both in Asia (11 RCTs, $n=1451, \mathrm{MD}-0.66$ days, $95 \% \mathrm{CI}-1.07$ to -0.26 ) and in Europe (3 RCTs, $n=237, \mathrm{MD}-0.88$ days, $95 \% \mathrm{CI}-1.41$ to -0.36$)$, but Lactobacillus acidophilus had no effect on the duration of diarrhea in America ( $1 \mathrm{RCT}, n=77$, MD -0.65 days, $95 \% \mathrm{CI}-1.33$ to 0.03 ) (Figure S5). The test for subgroup differences suggested no significant difference $(p=0.78)$, meaning that geographic setting may not modify the treatment effect. However, an RCT, and a smaller group of participants, contributed to the America subgroup compared to the non-America subgroup. Overall, to establish whether there is an actual significant subgroup effect requires more trials.

\section{- Etiology}

Concerning etiology, limited data indicate that Lactobacillus acidophilus was effective in treating diarrhea due to rotavirus-positive $100 \%$ ( $1 \mathrm{RCT}, n=57, \mathrm{MD}-1.23$ days, $95 \% \mathrm{CI}-1.88$ to -0.58 ), rotavirus-positive $1 \% \sim 99 \%$ (6 RCTs, $n=718$, MD -0.79 days, $95 \% \mathrm{CI}-1.43$ to -0.15 ) and unknown causes ( $8 \mathrm{RCTs}, n=990, \mathrm{MD}-0.91$ days, $95 \% \mathrm{CI}-1.24$ to -0.58 ) (Figure S6). The test for subgroup differences suggested no significant difference between groups $(p=0.60)$. However, each subgroup had a very small number of trials and individuals, making it difficult to draw clear conclusions.

\section{- Sensitivity Analyses}

Pre-planned subgroup analyses based on trial methodological quality were carried out. Statistically significant between the studies, heterogeneity persisted in subgroup analyses, suggesting that the outcomes were not sensitive to the studies' methodological quality, and that the outcomes were not sensitive to the classification according to the quality of methodology (Figures S7-S10). 


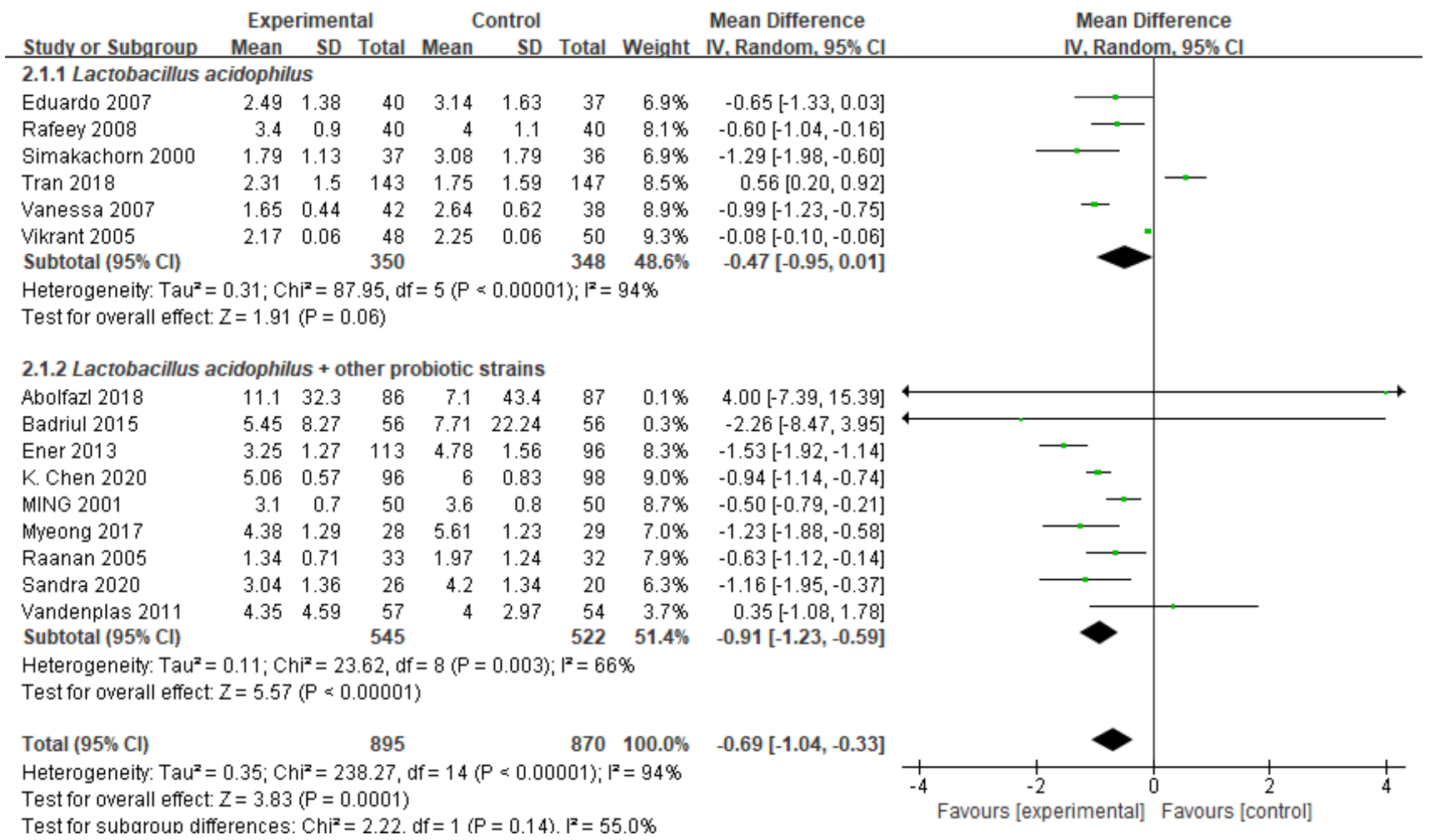

Figure 2. Forest plot of randomized controlled trials of Lactobacillus acidophilus vs. control in acute gastroenteritis. Effect on duration of diarrhea (days). Probiotic species.

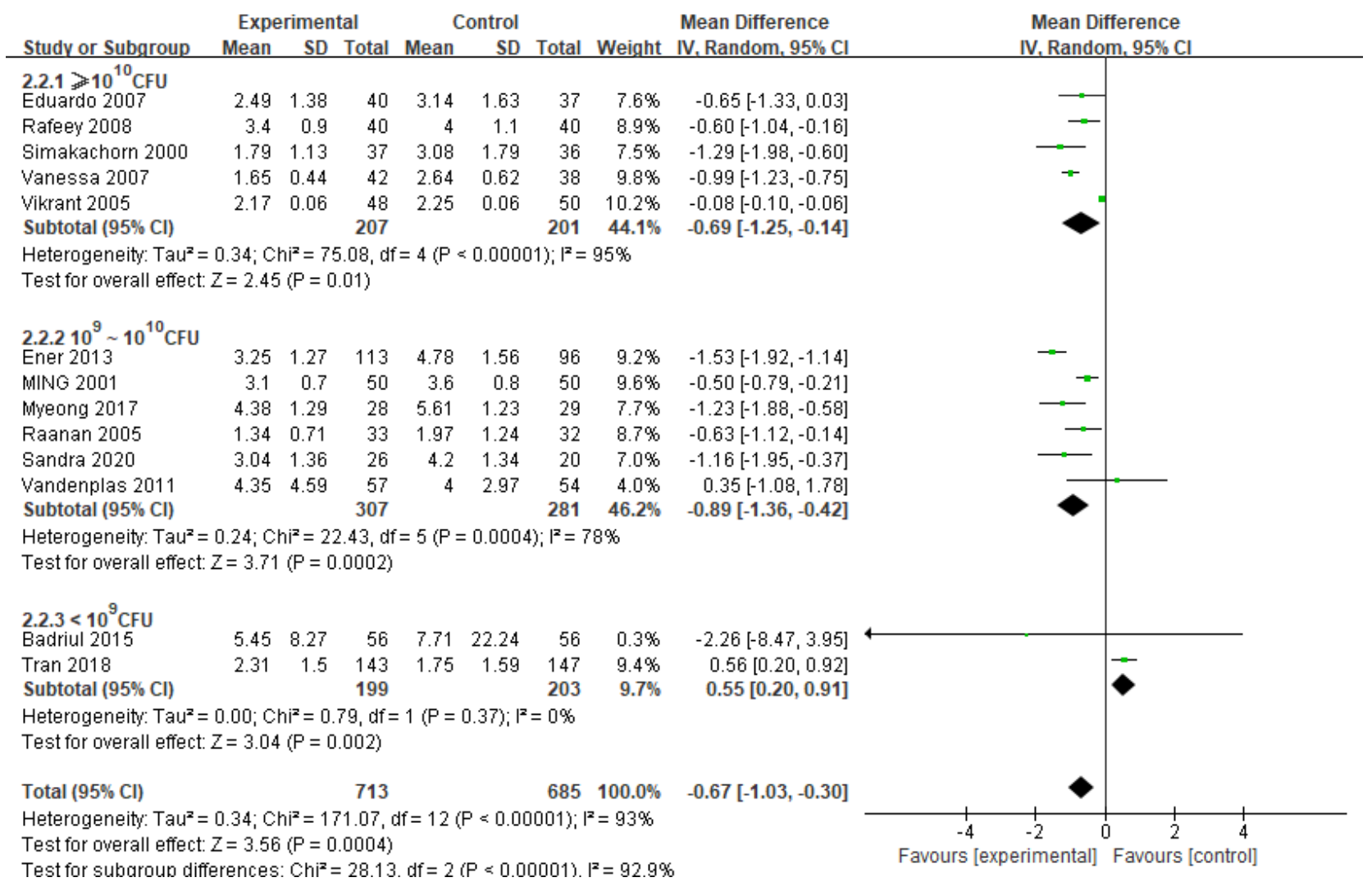

Figure 3. Forest plot of randomized controlled trials of Lactobacillus acidophilus vs. control in acute gastroenteritis. Effect on duration of diarrhea (days). Dose. 


\subsubsection{Diarrhea Frequency}

The included trials often reported diarrhea frequency. The studies showed a reduction in the frequency of diarrhea for those treated with Lactobacillus acidophilus compared with controls at all time intervals; however, it was just statistically significant at day 3 (4 RCTs, $n=594, \mathrm{MD}-0.61$ days, $95 \% \mathrm{CI}-1.00$ to -0.23 ) (Figure S11).

\section{Discussion}

This analysis was based on 15 trials, including 1765 children with acute gastroenteritis randomly assigned to Lactobacillus acidophilus or placebo. The present meta-analysis of Lactobacillus acidophilus for treating acute gastroenteritis in children substantially expanded on previous meta-analyses [30].

The addition of Lactobacillus acidophilus to standard rehydration therapy compared with placebo or no treatment was associated with a reduced duration of diarrhea by approximately $17 \mathrm{~h}$ (0.69 days). A subset of patients that was more likely to benefit included subjects treated with an effective daily dose of Lactobacillus acidophilus of $\geq 10^{9} \mathrm{CFU} /$ day. Lactobacillus acidophilus had the same effect when used in European countries and Asian countries. Limited evidence suggests that Lactobacillus acidophilus was more effective in treating diarrhea of rotavirus origin. Only a small number of studies looked at the impact of Lactobacillus acidophilus supplementation on the frequency of diarrheal episodes in children. While diarrhea frequency was decreased in general, it was statistically and clinically significant only on day 3, indicating that the optimum benefit of Lactobacillus acidophilus in lowering diarrhea frequency may be attained at $72 \mathrm{~h}$ after the intervention begins.

However, caution is needed when interpreting these results, as two included studies $[20,22]$ did not report the dose of each probiotic strain in the intervention; besides this dose issue, the course of acute gastroenteritis could also affect the efficacy of the probiotic. We think this is the most comprehensive analysis of this probiotic strain that has been conducted to date, and we hope that our findings will be especially useful to decision-makers. Because of inaccuracy, which was a key problem influencing confidence, and because there was considerable unexplained variability across studies, many component-effect estimates might be reinforced by further data, despite the large amount of data analyzed.

The present study would benefit from clarification of the uncertainties, for example, around the supplementation of zinc. Clinical studies have consistently observed an association between zinc deficiency and acute diarrhea in early childhood [31]. For babies and children suffering from severe diarrhea in resource-limited areas, the World Health Organization (WHO) advises that they receive zinc supplements [32]. Zinc supplementation was also observed to shorten the average duration of acute diarrhea in children in a meta-analysis [33]. However, in a network meta-analysis of randomized and quasirandomized trials comparing interventions for acute diarrhea and gastroenteritis, zinc was not effective in reducing the duration of diarrhea in children in high-income countries [9]. There is still controversy around the actual efficacy of zinc for the management of acute diarrhea. In our study, zinc was added to three RCTs $[11,22,24]$ and was absent in the other RCTs. Therefore, it remains to be established if additional zinc provision will benefit acute diarrhea and will have any influence on analysis results. The included trials covered most children. Children from both developing and developed countries aged from 1 month to 12 years old participated. However, there were some issues with the under-reporting of trial details in this area. We were missing data from many of the studies included, with data on specific population characteristics (e.g., outcomes in different ages, feeding patternbreastfeeding, or complementary feeding) particularly poorly reported. Trials need to be subdivided in terms of their goals and objectives. The microbiome is very different between individuals; this is true not only for viral species but also for tribes and individual strains. For example, a study which applied metagenomic analysis to fecal samples from infants and their mothers showed that the samples clustered according to age, and demonstrated that the 12-month-old infant samples were most similar to the mothers. There was a more complex and less heterogeneous community as a function of time. The rising complexity 
was also supported by increased numbers of microbial genomes identified in the older infants [34]. It would be of benefit to stratify study populations further if large groups are included, such as stratifying children from birth to 4 months, 4 to 12 months, and over 12 months, to allow for better analysis as well as to determine whether the gastrointestinal tracts of children are distinctive at the initiation of trials. Research has shown that human milk contains, not only macro- and micronutrients, but also living cells, growth factors, and immunoprotective substances $[35,36]$. The use of human milk lowers the risk of gastrointestinal illness compared with complimentary food or milk powder, and it may be of particular importance when evaluating the impact of interventions on groups with different diets. Due to a lack of reporting regarding population characteristics, we were unable to determine with any certainty whether population characteristics influenced the comparative effectiveness of the interventions evaluated. Individual participant data (particularly for population characteristics) meta-analysis, or network meta-analysis using original datasets, may be the best way to investigate this in the future.

Moreover, we believe that the effects of probiotic use will vary across viral causes, but there is insufficient etiological data to support this. In our study, the rotavirus infection status reported for only seven trials [10,12,16,23,27-29], of which only one trial [12] tested all participants for rotavirus infection. Furthermore, owing to missing data on rotavirus vaccination status in the included trials, it is unclear whether rotavirus vaccine moderates probiotic effectiveness.

A probiotic strain is considered to have specificity. Thus, a probiotic product used in clinical trials should record the genus, species, and strain designation clearly; all trials included performed well in this aspect.

The included trials also need to collect more outcome data, such as duration of vomiting, daycare absenteeism, the rate of household transmission and cost-effectiveness, as these may affect final decisions on curative probiotic supplementation for children.

\subsection{Quality of the Evidence}

Given that the number of RCTs and participants was not impressive, the quality of evidence supporting key findings was moderate to low. The majority of our component effect estimates were downgraded by one or two levels due to imprecision, owing to the different definitions of acute gastroenteritis/diarrhea, the different operational standards, and the different definitions of the reported outcomes among trials. For example, the different time of the initiation of probiotic administration (duration of diarrhea less than $48 \mathrm{~h}$, or $72 \mathrm{~h}$ ) or the different definition of diarrhea cure (stool met the Bristol criteria or not) caused imprecision and inconsistency as defined by GRADE.

More than a half of the trials adequately generated their randomization sequence (e.g., sealed envelopes, computer-generated randomization lists, random permuted blocks); almost $73 \%$ of the trials adequately concealed allocation; $80 \%$ of the trials blinded all the parties involved, e.g., participants, study personnel and outcome assessors; and 93\% provided complete outcome data. Eight included trials were considered to be at low risk of bias. In terms of certainty of evidence on outcomes, we have moderate confidence in effect estimates for the duration of diarrhea based on GRADE assessments.

\subsection{Strengths and Limitations}

The findings of this study, taken as a whole, contradict the findings of several of the low-risk bias studies that were included. There were a variety of factors that contributed to these variances. For example, the number of participants in Badriul 2015 [11] was 112, Myeong 2017 [12] was 57, and Vikrant 2005 [29] was 98; all the participant groups were not large enough, thus, the findings, whether positive or negative, might be (non)significant by chance only.

In 2019, a meta-analysis by Hania et al. [37] concluded that Lactobacillus rhamnosus GG can reduce the duration of diarrhea when used at a daily dose $\geq 10^{10} \mathrm{CFU}$ (11 RCTs, $n=2764$, MD -0.83 days, $95 \% \mathrm{CI}-1.17$ to -0.49 ) or $<10^{10} \mathrm{CFU}(4 \mathrm{RCTs}, n=1056$, MD -0.92 days, 
$95 \%$ CI -1.83 to -0.02 ). This finding contradicted the results of Badriul 2015 [11], whose intervention included the probiotic strains Lactobacillus acidophilus $0.1 \times 10^{9} \mathrm{CFU} /$ day and Lactobacillus rhamnosus GG $1.9 \times 10^{9} \mathrm{CFU} /$ day. Further studies are needed to assess the effect of probiotic mixtures of Lactobacillus acidophilus and Lactobacillus rhamnosus GG. The lack of an effect of Lactobacillus acidophilus in Badriul 2015 [11] and Tran 2018 [10] studies may also be explained by the fact that, in contrast to other studies, a low daily dose of the probiotic $\left(<10^{9} \mathrm{CFU}\right)$ was administered. Lactobacillus acidophilus was not beneficial in treating children with acute watery diarrhea, probably because the minimum effective concentration of Lactobacillus acidophilus was not reached.

In 2014, guidelines for the management of acute gastroenteritis in children published by the European Society for Paediatric Gastroenterology, Hepatology and Nutrition [3] concluded that 'the use of the probiotic Lactobacillus rhamnosus GG and Saccharomyces boulardii may be considered in the management of children with acute gastroenteritis in addition to rehydration therapy.' Two large, well-conducted meta-analyses showed that Lactobacillus rhamnosus GG and Saccharomyces boulardii can be effective in treating children with acute gastroenteritis $[37,38]$. However, the European Medicines Agency (EMA) has issued a recent warning about a potential risk of fungaemia caused by Saccharomyces boulardii in seriously ill or immunocompromised patients, and it has recommended that Saccharomyces boulardii be used with caution due to the possibility of airborne contamination. In conclusion, because of safety concerns associated with the use of Saccharomyces boulardii, we recommend the probiotic strains Lactobacillus acidophilus and Lactobacillus rhamnosus GG to children with acute gastroenteritis, whether the probiotic strains are used in combination or alone.

While the methodology of this systematic review was robust, the conclusions are mostly constrained by the number of papers that were accessible. There are some limitations to this review. The first is the possibility of bias in some of the trials that have been included.

Second, unexplainable heterogeneity between individual trials is a significant limitation. Clinical variations and methodological issues are among the factors contributing to the heterogeneity. On clinical differences, some subgroup analyses were conducted to investigate whether factors, such as dose, setting or etiology, modified the effect of the treatment. The operational standards for each trial were not uniform, which caused other kinds of clinical differences, such as inconsistency in zinc supplementation, different definitions of diarrhea, different durations of symptoms before the intervention, and different definitions of the reported outcomes. Such diversity and inconsistency in the outcomes, combined with the lack of standardized definitions, pose a challenge in meta-analyses and should be taken into account when interpreting the findings. On methodological issues, certain subgroup analyses were carried out in order to determine if the quality of the methodology affected the treatment impact. Overall, while some of the analyses revealed a statistically significant subgroup effect and high heterogeneity between findings within each group, the small number of trials and participants contributing to each subgroup resulted in uncertainty about whether these subgroup differences matter.

Due to the differences in clinical characteristics, or the fact that some outcomes were evaluated in only a subset of trials with a limited number of participants, meta-regression is underpowered to detect small associations or diversities; meta-regression results should hence be interpreted with caution. For example, in our study, the finding that etiology had no influence on treatment effects may have been due to the limited power with which we were able to identify them.

\section{Conclusions}

The epidemiology literature is becoming saturated with probiotic and gastrointestinal disease studies, and the use of probiotic strains is being developed more meticulously. In summary, our findings suggest Lactobacillus acidophilus treatment of acute gastroenteritis in children is effective when administered at a daily dosage of $\geq 10^{9} \mathrm{CFU} /$ day. But there are differences in the efficacy and acceptability profiles across Lactobacillus acidophilus, Lac- 
tobacillus rhamnosus and Saccharomyces boulardii, especially safety issues with the use of Saccharomyces boulardii. We recommend the probiotic strains Lactobacillus acidophilus and Lactobacillus rhamnosus for children with acute gastroenteritis, whether the probiotic strains are used in combination or alone. We hope that this analysis contributes a helpful perspective to aid in these decisions. Whether probiotic recommendations for acute gastroenteritis in children should be tailored based on susceptibility factors (for example, age, feeding pattern, whether vaccinated against rotavirus) remains unanswered and will be important to advance knowledge in the field.

Supplementary Materials: The following supporting information can be downloaded at: https:/ / www.mdpi.com/article/10.3390/nu14030682/s1, Table S1: Search strategy, Table S2: Characteristics of the included studies, Table S3: Characteristics of the excluded trials, Table S4: GRADE analysis for selected outcomes. Figure S1: Risk of bias in the included studies, Figure S2: Funnel plot, Figure S3: Lactobacillus acidophilus vs. control. Duration of diarrhea. Bacterial species (individual probiotic strains Lactobacillus acidophilus vs. Lactobacillus acidophilus + Bifidobacterium vs. Lactobacillus acidophilus + Lactobacillus rhamnosus vs. Lactobacillus acidophilus + other probiotic strains), Figure S4: Lactobacillus acidophilus vs. control. Duration of diarrhea. Dose of Lactobacillus acidophilus in individual probiotic strains studies (high dose $\left[\geq 10^{10} \mathrm{CFU} /\right.$ day $]$ vs. lower dose $\left[<10^{10} \mathrm{CFU} /\right.$ day $]$ ), Figure S5: Lactobacillus acidophilus vs. control. Duration of diarrhea. Setting (studies carried out in geographical areas Asia vs. Europe vs. America), Figure S6: Lactobacillus acidophilus vs. control. Duration of diarrhea. Etiology of diarrhea (rotavirus-positive 100\% vs. rotavirus-positive 1\% 99\% vs. unknown etiology), Figure S7: Lactobacillus acidophilus vs. control. Methodology. Randomization, Figure S8: Lactobacillus acidophilus vs. control. Methodology. Allocation concealment, Figure S9: Lactobacillus acidophilus vs. control. Methodology. Blinding, Figure S10: Lactobacillus acidophilus vs. control. Methodology. Incomplete outcome data, Figure S11: Lactobacillus acidophilus vs. control. Frequency of stools.

Author Contributions: Conceptualization H.C., L.Z.; methodology X.Z.; formal analysis C.T.; investigation X.L.; writing-original draft preparation H.C. and Y.M.; writing-review and editing X.Z.; supervision L.Z. All authors have read and agreed to the published version of the manuscript.

Funding: This work was supported by Beijing Municipal Natural Science Foundation (L202042), and National Science Foundation (82173894).

Institutional Review Board Statement: Not applicable.

Informed Consent Statement: Not applicable.

Data Availability Statement: Data sharing not applicable.

Acknowledgments: We would like to acknowledge the valuable contribution of the authors of the papers included in the present systematic review and meta-analysis, who very kindly provided additional data regarding their papers.

Conflicts of Interest: The authors declare no conflict of interest.

\section{References}

1. World Health Organization. Diarrhoeal Disease Fact Sheet. Available online: http://www.who.int/mediacentre/factsheets/fs3 30/en (accessed on 25 February 2021).

2. $\quad$ Dhingra, U.; Kisenge, R.; Sudfeld, C.R.; Dhingra, P.; Somji, S.; Dutta, A.; Bakari, M.; Deb, S.; Devi, P.; Liu, E. Lower-Dose Zinc for Childhood Diarrhea-A Randomized, Multicenter Trial. N. Engl. J. Med. 2020, 383, 1231-1241. [CrossRef] [PubMed]

3. Guarino, A.; Ashkenazi, S.; Gendrel, D.; Lo Vecchio, A.; Shamir, R.; Szajewska, H. European Society for Pediatric Gastroenterology, Hepatology, and Nutrition/European Society for Pediatric Infectious Diseases evidence-based guidelines for the management of acute gastroenteritis in children in Europe: Update 2014. J. Pediatr. Gastroenterol. Nutr. 2014, 59, 132-152. [CrossRef] [PubMed]

4. Lorca, G.L.; Raya, R.R.; Taranto, M.P.; Valdez de, G.F. Adaptive acid tolerance response in Lactobacillus acidophilus. Biotechnol. Lett. 1998, 20, 239-241. [CrossRef]

5. Borthakur, A.; Gill, R.K.; Tyagi, S.; Koutsouris, A.; Alrefai, W.A.; Hecht, G.A.; Ramaswamy, K.; Dudeja, P.K. The probiotic Lactobacillus acidophilus stimulates chloride/hydroxyl exchange activity in human intestinal epithelial cells. J. Nutr. 2008, 138, 1355-1359. [CrossRef] [PubMed]

6. Azevedo, M.S.; Zhang, W.; Wen, K.; Gonzalez, A.M.; Saif, L.J.; Yousef, A.E.; Yuan, L. Lactobacillus acidophilus and Lactobacillus reuteri modulate cytokine responses in gnotobiotic pigs infected with human rotavirus. Benef. Microbes. 2012, 3, 33-42. [CrossRef] [PubMed] 
7. Liévin-Le Moal, V. A gastrointestinal anti-infectious biotherapeutic agent: The heat-treated Lactobacillus LB. Therap Adv Gastroenterol. 2016, 9, 57-75. [CrossRef]

8. Seth, A.; Yan, F.; Polk, D.B.; Rao, R.K. Probiotics ameliorate the hydrogen peroxide-induced epithelial barrier disruption by a PKC- and MAP kinase-dependent mechanism. Am. J. Physiol. Gastrointest. Liver Physiol. 2008, 294, G1060-G1069. [CrossRef]

9. Florez, I.D.; Veroniki, A.A.; Al Khalifah, R.; Yepes-Nuñez, J.J.; Sierra, J.M.; Vernooij, R.W.M.; Acosta-Reyes, J.; Granados, C.M.; Pérez-Gaxiola, G.; Cuello-Garcia, C.; et al. Comparative effectiveness and safety of interventions for acute diarrhea and gastroenteritis in children: A systematic review and network meta-analysis. PloS ONE 2018, 13, e0207701. [CrossRef]

10. Hong Chau, T.T.; Minh Chau, N.N.; Hoang Le, N.T.; Chung The, H.; Voong Vinh, P.; Nguyen To, N.T.; Ngoc, N.M.; Tuan, H.M.; Chau Ngoc, T.L.; Kolader, M.E.; et al. A Double-blind, Randomized, Placebo-controlled Trial of Lactobacillus acidophilus for the Treatment of Acute Watery Diarrhea in Vietnamese Children. Pediatr. Infect Dis. J. 2018, 37, 35-42. [CrossRef]

11. Hegar, B.; Waspada, I.M.; Gunardi, H.; Vandenplas, Y. A double blind randomized trial showing probiotics to be ineffective in acute diarrhea in Indonesian children. Indian J. Pediatr. 2015, 82, 410-414. [CrossRef]

12. Park, M.S.; Kwon, B.; Ku, S.; Ji, G.E. The Efficacy of Bifidobacterium longum BORI and Lactobacillus acidophilus AD031 Probiotic Treatment in Infants with Rotavirus Infection. Nutrients 2017, 9, 887. [CrossRef] [PubMed]

13. McMaster University and Evidence Prime. GRADEpro GDT, Version updated 2017; McMaster University and Evidence Prime: Hamilton, ON, Canada, 2017.

14. Higgins, J.P.; Thomas, J.; Chandler, J.; Cumpston, M.; Li, T.; Page, M.J.; Welch, V.A. (Eds.) Cochrane Handbook for Systematic Reviews of Interventions. Available online: https:// training.cochrane (accessed on 25 February 2021).

15. Egger, M.; Davey Smith, G.; Schneider, M.; Minder, C. Bias in meta-analysis detected by a simple, graphical test. BMJ (Clin. Res.) 1997, 315, 629-634. [CrossRef] [PubMed]

16. Salazar-Lindo, E.; Figueroa-Quintanilla, D.; Caciano, M.I.; Reto-Valiente, V.; Chauviere, G.; Colin, P. Effectiveness and safety of Lactobacillus LB in the treatment of mild acute diarrhea in children. J. Pediatr. Gastroenterol. Nutr. 2007, 44, 571-576. [CrossRef] [PubMed]

17. Vandenplas, Y.; De Hert, S.G. Randomised clinical trial: The synbiotic food supplement Probiotical vs. placebo for acute gastroenteritis in children. Aliment Pharmacol. Ther. 2011, 34, 862-867. [CrossRef]

18. Wan, X.; Wang, W.; Liu, J.; Tong, T. Estimating the sample mean and standard deviation from the sample size, median, range and/or interquartile range. BMC Med. Res. Methodol. 2014, 14, 135. [CrossRef]

19. McGrath, S.; Zhao, X.; Steele, R.; Thombs, B.D.; Benedetti, A. DEPRESsion Screening Data (DEPRESSD) Collaboration. Estimating the sample mean and standard deviation from commonly reported quantiles in meta-analysis. Stat. Methods Med. Res. 2020, 30, 962280219889080.

20. Kasiri, K.A.; Khoshdel, A.; Salehi, S.; Sedehi, M. Effect of probiotics in the treatment of acute noninflammatory diarrhea in hospitalized children aged 2-10 years. Int. J. Pharm. Investig. 2018, 8, 200-204. [CrossRef]

21. Dinleyici, E.C.; Dalgic, N.; Guven, S.; Ozen, M.; Kara, A.; Arica, V.; Metin-Timur, O.; Sancar, M.; Kurugo, 1.Z.; Tanir, G.; et al. The effect of a multispecies synbiotic mixture on the duration of diarrhea and length of hospital stay in children with acute diarrhea in Turkey: Single blinded randomized study. Eur. J. Pediatr. 2013, 172, 459-464. [CrossRef]

22. Chen, K.; Xin, J.; Zhang, G.; Xie, H.; Luo, L.; Yuan, S.; Bu, Y.; Yang, X.; Ge, Y.; Liu, C. A combination of three probiotic strains for treatment of acute diarrhoea in hospitalised children: An open label, randomised controlled trial. Benef Microbes. 2020, 11, 339-346. [CrossRef]

23. Lee, M.C.; Lin, L.H.; Hung, K.L.; Wu, H.Y. Oral bacterial therapy promotes recovery from acute diarrhea in children. Acta Paediatr Taiwan 2001, 42, 301-305.

24. Shamir, R.; Makhoul, I.R.; Etzioni, A.; Shehadeh, N. Evaluation of a diet containing probiotics and zinc for the treatment of mild diarrheal illness in children younger than one year of age. J. Am. Coll. Nutr. 2005, 24, 370-375. [CrossRef] [PubMed]

25. Rafeey, M.; Ostadrahimi, A.; Boniadi, M.; Ghorashi, Z.; Alizadeh, M.M.; Hadafey, V. Lactobacillus acidophilus yogurt and supplement in children with acute diarrhea: A clinical trial. Res. J. Med. Sci. 2008, 2, 13-18.

26. Kluijfhout, S.; Trieu, T.V.; Vandenplas, Y. Efficacy of the Probiotic Probiotical Confirmed in Acute Gastroenteritis. Pediatr. Gastroenterol. Hepatol. Nutr. 2020, 23, 464-471. [CrossRef] [PubMed]

27. Simakachorn, N.; Pichaipat, V.; Rithipornpaisarn, P.; Kongkaew, C.; Tongpradit, P.; Varavithya, W. Clinical evaluation of the addition of lyophilized, heat-killed Lactobacillus acidophilus LB to oral rehydration therapy in the treatment of acute diarrhea in children. J. Pediatr. Gastroenterol. Nutr. 2000, 30, 68-72. [CrossRef]

28. Lievin-Le Moal, V.; Sarrazin-Davila, L.E.; Servin, A.L. An experimental study and a randomized, double-blind, placebo-controlled clinical trial to evaluate the antisecretory activity of Lactobacillus acidophilus strain LB against nonrotavirus diarrhea. Pediatrics 2007, 120, 795-803. [CrossRef]

29. Khanna, V.; Alam, S.; Malik, A.; Malik, A. Efficacy of tyndalized Lactobacillus acidophilus in acute diarrhea. Indian J. Pediatr. 2005 72, 935-938. [CrossRef]

30. Szajewska, H.; Ruszczyński, M.; Kolaček, S. Meta-analysis shows limited evidence for using Lactobacillus acidophilus LB to treat acute gastroenteritis in children. Acta Paediatr. 2014, 103, 249-255. [CrossRef]

31. Black, R.E.; Victora, C.G.; Walker, S.P.; Bhutta, Z.A.; Christian, P.; Onis, M.; Ezzati, M.; Grantham-McGregor, S.; Katz, J.; Martorell, R.; et al. Maternal and child undernutrition and overweight in low-income and middle-income countries. Lancet (Lond. Engl.) 2013, 382, 427-451. [CrossRef] 
32. World Health Organization: Zinc Supplementation in the Management of Diarrhoea. Available online: https://www.who.int/ elena/titles/zinc_diarrhoea/en/ (accessed on 11 February 2019).

33. Lazzerini, M.; Wanzira, H. Oral zinc for treating diarrhoea in children. Cochrane Database Syst. Rev. 2016, 12, Cd005436. [CrossRef]

34. Bäckhed, F.; Roswall, J.; Peng, Y.; Feng, Q.; Jia, H.; Kovatcheva-Datchary, P.; Li, Y.; Xia, Y.; Xie, H.; Zhong, H.; et al. Dynamics and Stabilization of the Human Gut Microbiome during the First Year of Life. Cell Host Microbe 2015, 10, 852. [CrossRef]

35. Andreas, N.J.; Kampmann, B.; Mehring Le-Doare, K. Human breast milk: A review on its composition and bioactivity. Early Hum. Dev. 2015, 91, 629-635. [CrossRef] [PubMed]

36. Ballard, O.; Morrow, A.L. Human milk composition: Nutrients and bioactive factors. Pediatr. Clin. N. Am. 2013, 60, 49-74. [CrossRef] [PubMed]

37. Szajewska, H.; Kołodziej, M.; Gieruszczak-Białek, D.; Skórka, A.; Ruszczyński, M.; Shamir, R. Systematic review with metaanalysis: Lactobacillus rhamnosus GG for treating acute gastroenteritis in children-A 2019 update. Aliment Pharmacol. Ther. 2019, 49, 1376-1384. [CrossRef] [PubMed]

38. Szajewska, H.; Kołodziej, M.; Zalewski, B.M. Systematic review with meta-analysis: Saccharomyces boulardii for treating acute gastroenteritis in children-a 2020 update. Aliment Pharmacol. Ther. 2020, 51, 678-688. [CrossRef] [PubMed] 